\title{
THE DEVELOPMENT OF FIBRE OPTIC MICROBEND SENSORS*
}

\author{
W $H$ G HORSTHUIS and $J$ H J FLUITMAN
}

Twente University of Technology, PO Box 2177500 AE Enschede (The Netherlands)

(Received June 10, 1982, accepted in revised form August 8, 1982)

\begin{abstract}
Investigations concerning the development of a fibre optic pressure sensor are reported The phenomenon of light power attenuation in a periodically deformed graded index fibre has been studied theoretically It appears that avalable theories are not suited to describe this attenuation process in the case of strong fibre deformations We therefore suggest an approach which takes account of a correlation length in such strong attenuation processes Experimental results, obtained with a newly developed test device, are given and are compared with this theory and with former results it is concluded that the attenuation process is understood qualitatively, but that further research is needed to obtain a more detaled description of the observed behaviour
\end{abstract}

\section{Introduction}

Durng the last decade there has been tremendous development in fibre optic technology, mainly intended for communication purposes But the inherent advantages of fibre optics could be of great importance for sensor applications as well The use of optical fibres ensures, for instance, an isolation between sensor and control electronics, thereby overcoming traditional problems such as grounding or the risks of sparks This makes fibre optic sensors suitable for applications in inflammable or explosive environments, for example, in petrochemical applications The very low attenuation of a fibre and its insensitivity to EM fields open possibilities for remote sensing Such a sensor could be integrated into a fibre optic communication system

Indeed the interest in fibre optic sensors is now quickly growing It has been shown that a multitude of sensors can be made using fibre optics [1] An interesting proposal is an acoustic sensor based on the effects which

* Based on a paper presented at the S \& A Symposium on force, pressure, displace ment and flow sensors, Enschede, The Netherlands, May $13-14,1982$, and published with kind permission of the S \& A Research Unit, Enschede, The Netherlands 


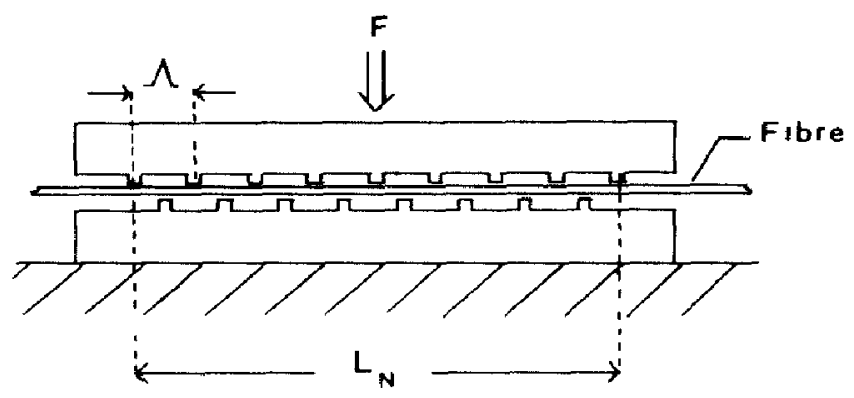

Fig 1 Fibre force sensor

occur if a fibre is deformed [2] If this deformation is periodic and the fibre is of a graded index type, a strong attenuation of the light power in the fibre may occur, even for very small deformation amplitudes

The fibre can be deformed as shown in Fig 1, where under the influence of an external force, the fibre undergoes microbending The fibre is pressed between two ridged plates with a total interaction length $L_{N}$, where $N$ is the number of ridges of one plate The deformation is considered to be harmonic with a period $\Lambda$ In our laboratory we studied the influences of several mechanical parameters on the microbend-induced attenuation

In the following sections we report our theoretical and experimental results concerning this subject The results are compared with our first findings which were reported in an earlier paper [3]

\section{Attenuation in a periodically deformed fibre}

If a fibre is deformed, guided modes may be scattered into radiant ones, which results in light-power loss This phenomenon has been extensively studied [4], since it is very harmful for communications purposes It has been demonstrated that for a certain value of the mechanically-induced spatial wavelength $\Lambda$, a resonant-like behaviour of the attenuation shows up [5] In a parabolic index fibre the guided modes are separated from each other by a constant difference in wavenumber This constant is given by [5]

$k_{c}=\sqrt{\frac{\overline{2 \Delta}}{r^{2}}}=\left(\frac{2 \pi}{\Lambda_{c}}\right)$

with $\Delta$ a parameter characterizing the index profile of the fibre core and $r$ the fibre core radius It happens to be the case that mode coupling is induced by a spatial deformation with $\Lambda=\Lambda_{c}$ In other words, if the applied $\Lambda$ fits the mode separation wavenumber $k_{\mathrm{c}}$, mode scattering will occur Since all guided modes are equally spaced, it turns out that if one guided mode can be scattered into another mode, all guided modes can be scattered into their neighbouring modes Because the modes with the highest wavenumber are 
partially scattered into radiant ones, a continuous energy flow into the radiant mode spectrum takes place, causing detectable light-power loss at the end of a fibre

If the power output is defined according to

$P=P_{0} \exp (-\alpha z)$

with $P$ the light-power output, $P_{0}$ the power input, $\alpha$ the loss coefficient and $z$ the longitudinal coordinate, first-order perturbation theory predicts a loss coefficient $\alpha$ proportional to [6]

$F(k)=\left|\frac{1}{\sqrt{L_{N}}} \int_{0}^{L_{N}} f(z) \exp (-t k z) \mathrm{d} z\right|^{2}$

which is the power spectrum of a mechanical deformation $f(z)$ along a fibre segment with length $L_{N}$

If we substitute a harmonic function for $f(z)$ we find

$F(k)=\frac{1}{4} d^{2} L_{N}\left|\frac{\sin \left(\left(k-k_{\mathrm{c}}\right) \frac{1}{2} L_{N}\right)}{\left(k-k_{\mathrm{c}}\right) \frac{1}{2} L_{N}}\right|^{2}$

with $d$ the amplitude of $f(z)$ The resonant-like behaviour at $k=k_{\mathrm{c}}$ is easily recognized in eqn (4)

It must be remembered that eqns (3) and (4) are results of first-order perturbation theory Since the losses that can be predicted with this theory and can be measured in practice are so large, one can have doubts about the validity of the formulae The most senous objection is, that in the case of large attenuation there can be no correlation of the light-power distribution over the total length of the deformed fibre So it is expected that in such a case $L_{N}$ can no longer appear as a parameter in the loss coefficient As a tentative extension of the theory to the case of large attenuation, we suggest replacing $L_{N}$ by a correlation length $L_{0}\left(<L_{N}\right)$, a procedure which is in some way analogous to that exploited in the calculation of losses in long fibres with random bends [6] $L_{0}$ might be identified with an effective mean free path of the light modes, or with the range within which the light power reaches a steady-state mode distribution

In ref 6 it is shown that a steady-state mode distribution is reached after the light has passed a characteristic length $L_{0}$ of fibre, independent of the mode distribution at the start In between, the power distribution can be expressed as a superposition of decaying components

$P(z, k)=\sum_{n=1}^{\infty} C_{n} G_{n}(k) \exp \left(-\sigma_{n} z\right)$

(we assume the fibre, apart from the bending loss, to be lossless) with $C_{n}$ determined by the initial power distribution In a steady state, all but the longest-lived components die out $\mathrm{A}$ criterion for $L_{0}$ may be found in comparing $\exp \left(-\sigma_{1} z\right)$ with $\exp \left(-\sigma_{2} z\right)$, for instance through the introduction of a coefficient $\beta$ in 
$\frac{\exp \left(-\sigma_{1} L_{0}\right)}{\exp \left(-\sigma_{2} L_{0}\right)}=\exp (\beta)$

An experimental estimate of $\beta$ will be obtained below

From Marcuse [6] we find $\sigma_{n} \sim u_{n}^{2}$, where the parameters $u_{n}$ are determined as the roots of the equation $J_{0}\left(u_{n}\right)=0$ So 1 follows that

$\frac{\sigma_{2}}{\sigma_{1}}=\frac{u_{2}^{2}}{u_{1}^{2}}=\frac{552^{2}}{240^{2}} \approx 5$

Substitution of this value in eqn (5) gives $L_{0}=\beta / 4 \sigma_{1}$ for the correlation length and with $\sigma_{1}=C F(k)$ (see ref [6],C is a constant) we find

$L_{0}=\frac{\beta}{4 C F(k)}$

for $k=k_{c}$ and $L_{N}$ subslituted by $L_{0}$, eqn (4) becomes $F(k)=d^{2} L_{0} / 4$, so that we ultimately find

$L_{0}=\left(\frac{\beta}{C}\right)^{1 / 2} \frac{1}{d}$

It is seen that the correlation length is inversely proportional to $d$ We can have the following situations

a) $L_{0}<L_{N}$ In this case the losses are determined by $L_{0}$ and for a piece of fibre with length $L_{N}$ (so $z=L_{N}$ ) we have, for $k=k_{\mathrm{c}}$

$\ln \left(P_{0} / P\right)=\sigma_{1} z=\frac{1}{4} \sqrt{\beta C} d L_{N}$

b) For $L_{0}>L_{N}$ and assuming a steady-state distribution at $z=0$ we find

$\ln \left(P_{0} / P\right)=\sigma_{1} z=\frac{C}{4} d^{2} L_{N}^{2}$

(Note that if a steady state already exists at $z=0$, it has no influence on the relevance of the correlation length)

c) At $L_{0}=L_{N}$ we define the 'turnover' from one behaviour into the

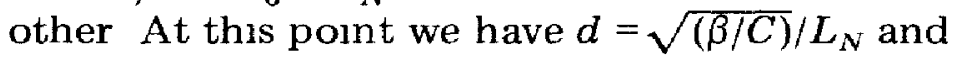

$\ln \left(P_{0} / P\right)=\sigma_{1} z=\beta / 4$

So it may be concluded that the loganthmic power loss, as a function of $d$, has an initial quadratic form and gradually turns into a linear function Next it can be stated that the deformation amplitude should be proportional to the force per ridge $d \sim F / N$ Since $L_{N} \sim N$, eqns (8) and (9) simply turn into

$$
\begin{array}{ll}
\ln \left(P_{\mathrm{o}} / P\right) \sim F^{2} & \left(L_{0}>L_{N}\right) \\
\ln \left(P_{\mathrm{o}} / P\right) \sim F & \left(L_{0}<L_{N}\right)
\end{array}
$$


irrespective of the number of ridges A second conclusion of our theory is that the spectral width of the power-loss function is determined by $L_{N}$ and independent of $d$ for small perturbations and by $L_{0} \sim 1 / d$ for large perturbations

\section{Expermmental set up}

In order to study the validity of the proposed theory, we had to set up experiments in which the different parameters could be varied independently In earlier investigations concerning the microbend attenuation, it became very clear that an extremely accurate mechanical system is needed in order to obtain reliable results [3] In our original experimental set up we experenced some major problems which are listed below

1) The plates between which the fibre is pressed must be perfectly alıgned The angle between the plates should be smaller than 1/1000 of a degree This requirement ensures that all the ridges of both plates touch the fibre simultaneously of course the plates themselves should have a perfect profile

2) In order to press the fibre, one of the plates must be movable This movement must not disturb the alignment of the two plates

3) The movement mentioned above should be frictionless in order to prevent unknown frictional forces and hysteresis

We designed a new measuring device which solved these problems Figures 2 to 5 show the device with some specific detals Figure 2 shows one

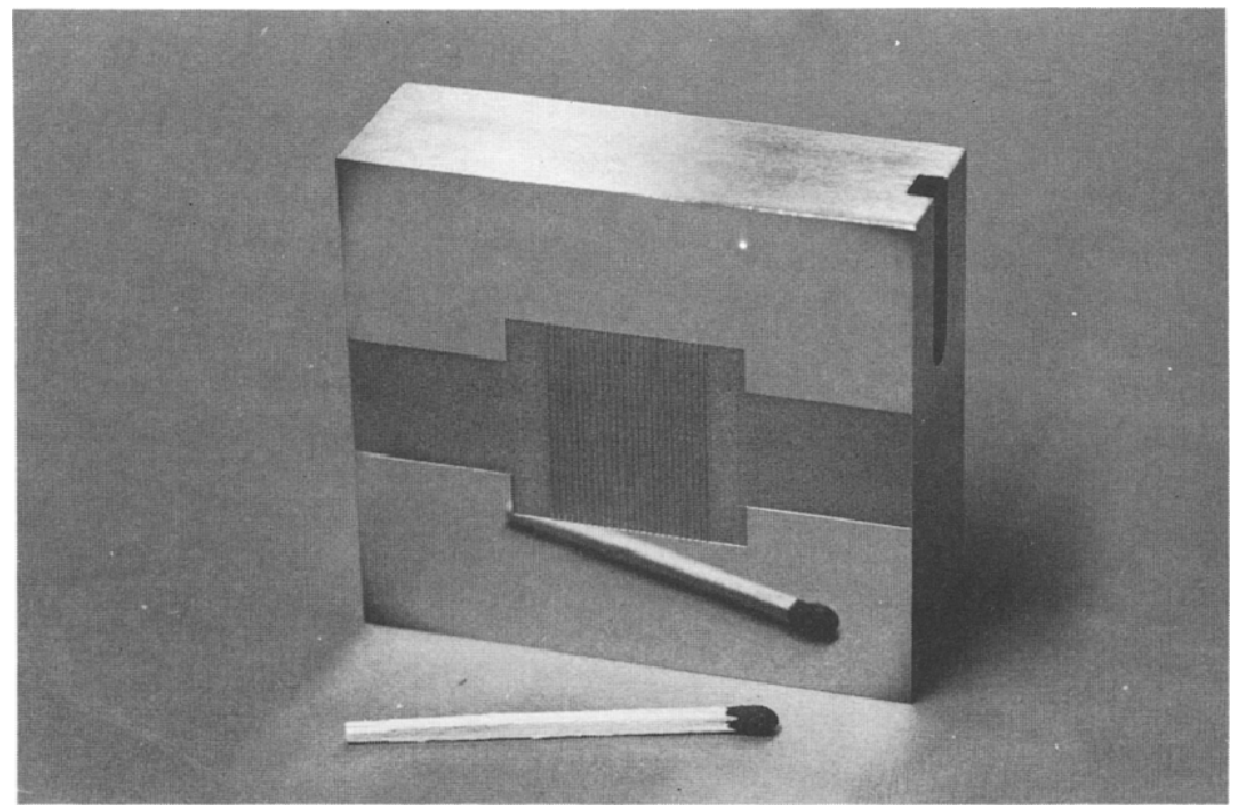

Fig 2 One of the ridged plates 


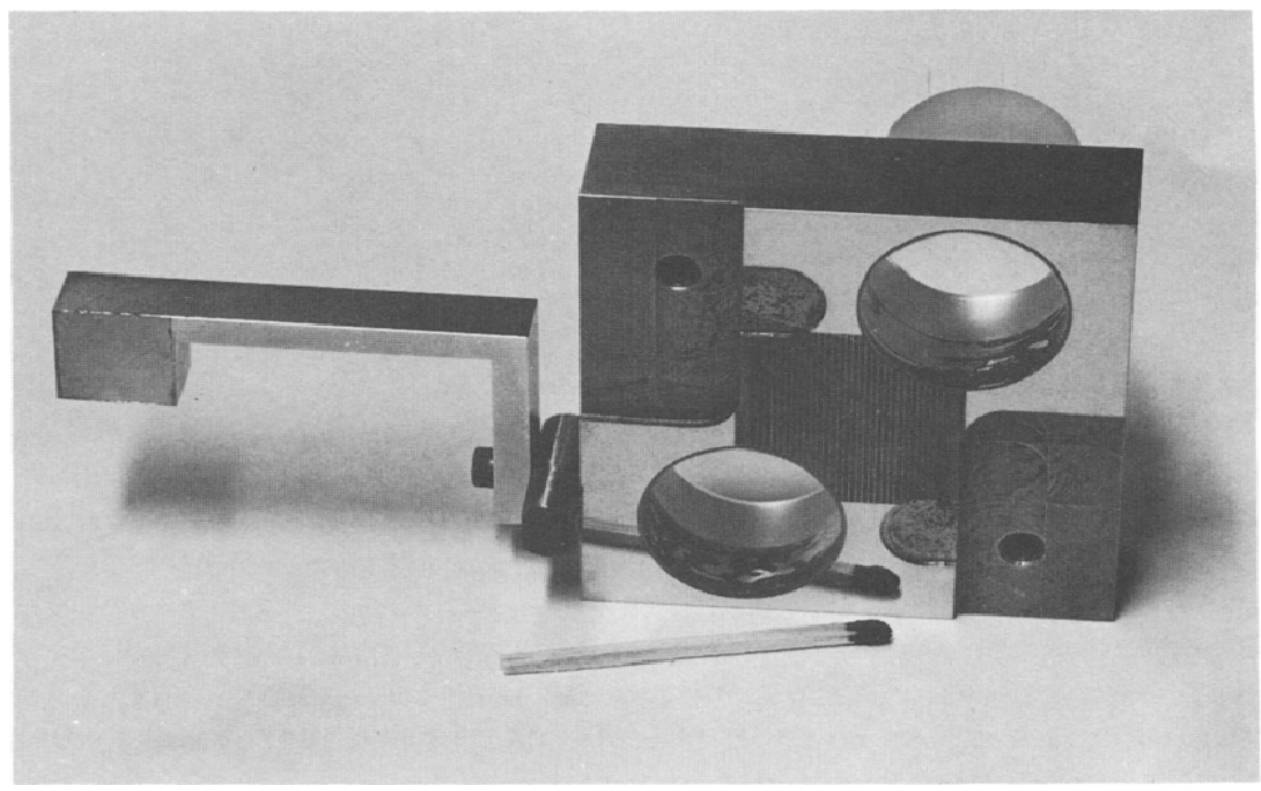

Fig 3 The fixed plate

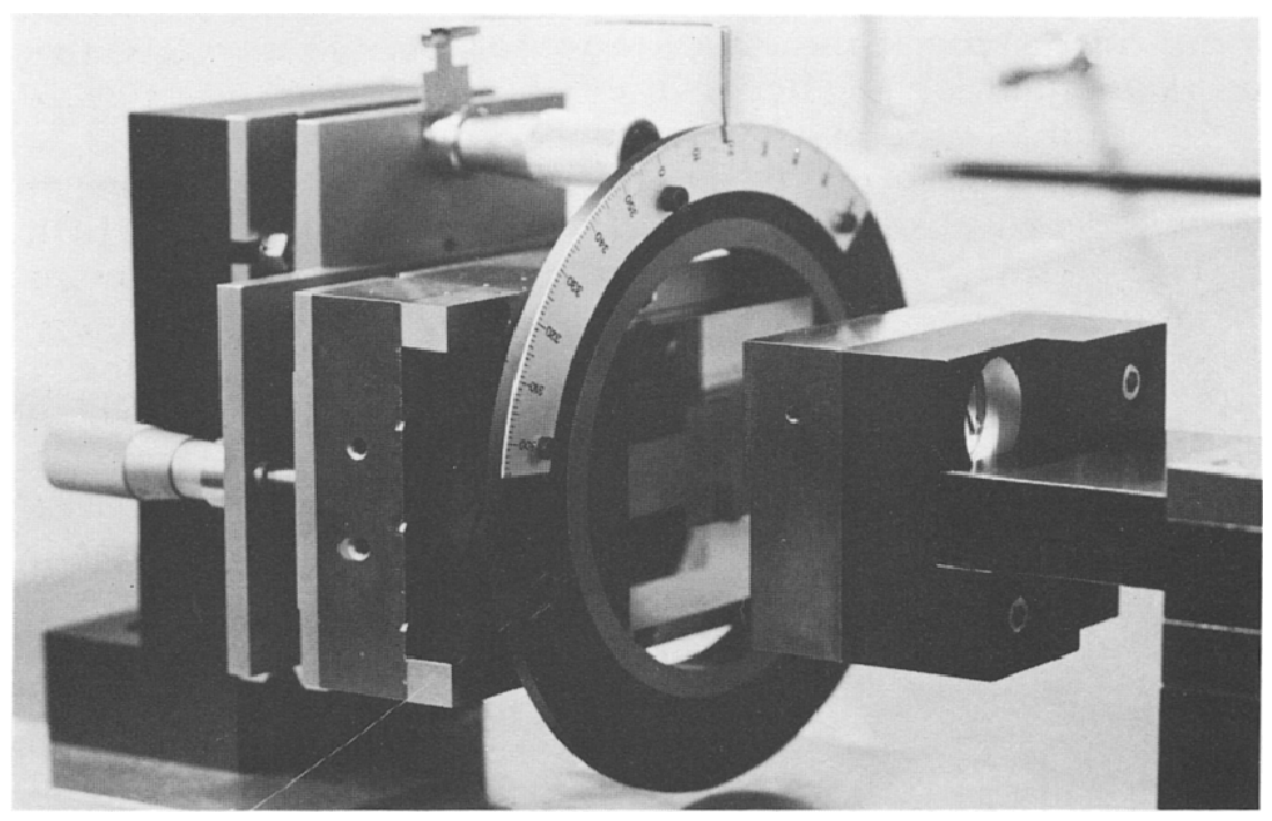

Fig \& The mounting of the plates

of the ridged plates, composed of hardened steel The profile in it is formed by means of spark erosion, and is polished afterwards 'There are seven plates like this, each consisting of a different number of ridges $4,8,12,16,20,24$ and 28 respectively The fibre is to be pressed between one of these plates and the plate shown in Fig 3, which consists of 29 ridges In addition it contains two interference glasses The glass surfaces are in one plane with the top of the ridges and the rest of the plate within $03 \mu \mathrm{m}$ 


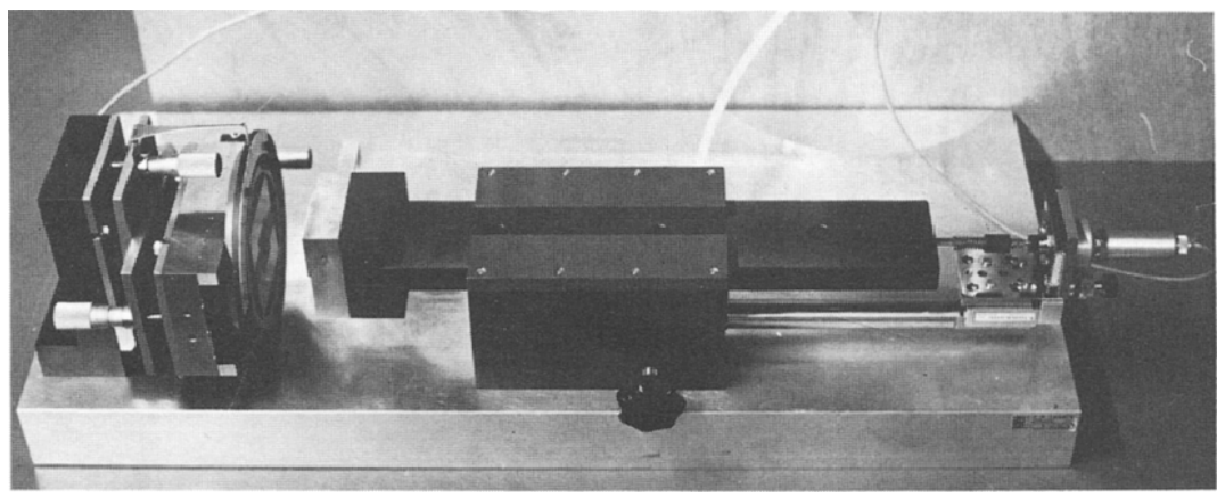

Fig 5 The complete mounted device

If this plate is pressed on one of the other plates (without a fibre in between), an interference pattern can be seen lookıng through the glasses from the rear The mounting of the removable plate can be adjusted in two perpendicular directions (see Fig 4) The adjustment is made using the two micrometers seen on the left The plates can be aligned exactly by observation of the interference pattern through the glasses of the fixed plate

Furthermore, Fig 4 shows the fibre which is glued on a ring This ring can rotate along the exchangeable plate This rotation changes the effective spatial wavelength according to $\Lambda=\sin (\phi) / S$, with $\phi$ the angle between fibre and ridges, and $S$ the pitch between two adjacent ridges

On the right side of Fig 4 the fixed plate is seen mounted on an air bearing axis The use of an air bearing ensures a frictionless movement which is perpendicular to the two plates Figure 5 shows the complete mounted device The force pressing on the fibre is induced by a micrometer and measured with a force transducer, both seen on the right of the device Finally the fibre deformation amplitude is measured with a proximity sensor which can be seen behind the fibre rotation ring

With the ald of this device we can measure the separate influences of three parameters on the microbend attenuation First, the number of bends by changing the plate, secondly the spatial wavelength by rotating the ring with the fibre and thirdly the applied force by the micrometer

The fibre we used in the experiments was a graded index fibre (Philips GR 2875) with a core diameter of $50 \mu \mathrm{m}$, a cladding diameter of $100 \mu \mathrm{m}$, $\Delta=0016$ and therefore $\Lambda_{c}=088 \mathrm{~mm}$ (see eqn (1)) This fibre has a $2 \mu \mathrm{m}$ primary coating of PAI which acts as a continuous modestripper Light from a pulsed IRED (Siemens LD 261) was directly injected into this fibre The output was detected with a PIN diode (Siemens BPX 65) The electronic circuitry could detect $005 \%$ intensity variations of the fibre output

\section{Experumental results}

The first measurements were made on the relation between the spatial wavelength $\Lambda$ and the induced attenuation for every avalable number of 

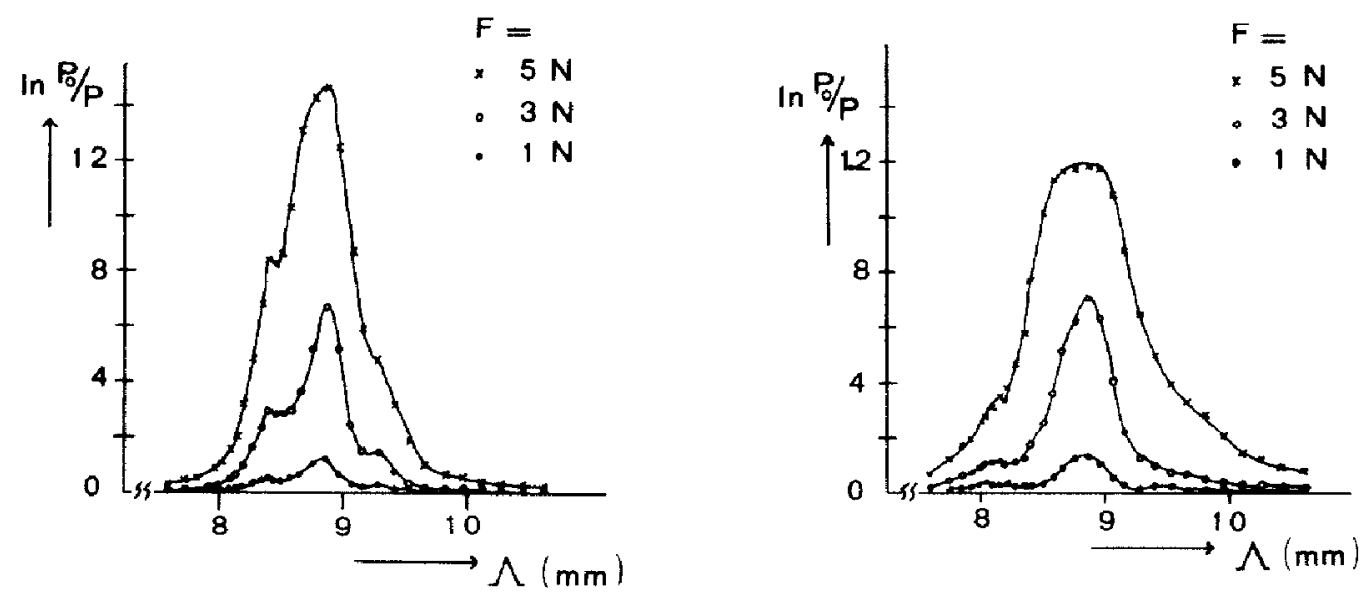

Fig 6 Attenuation versus spatial wavelength at three values of the applied force $N=28$ Fig 7 Attenuation versus spatial wavelength at three values of the applied force $N=16$
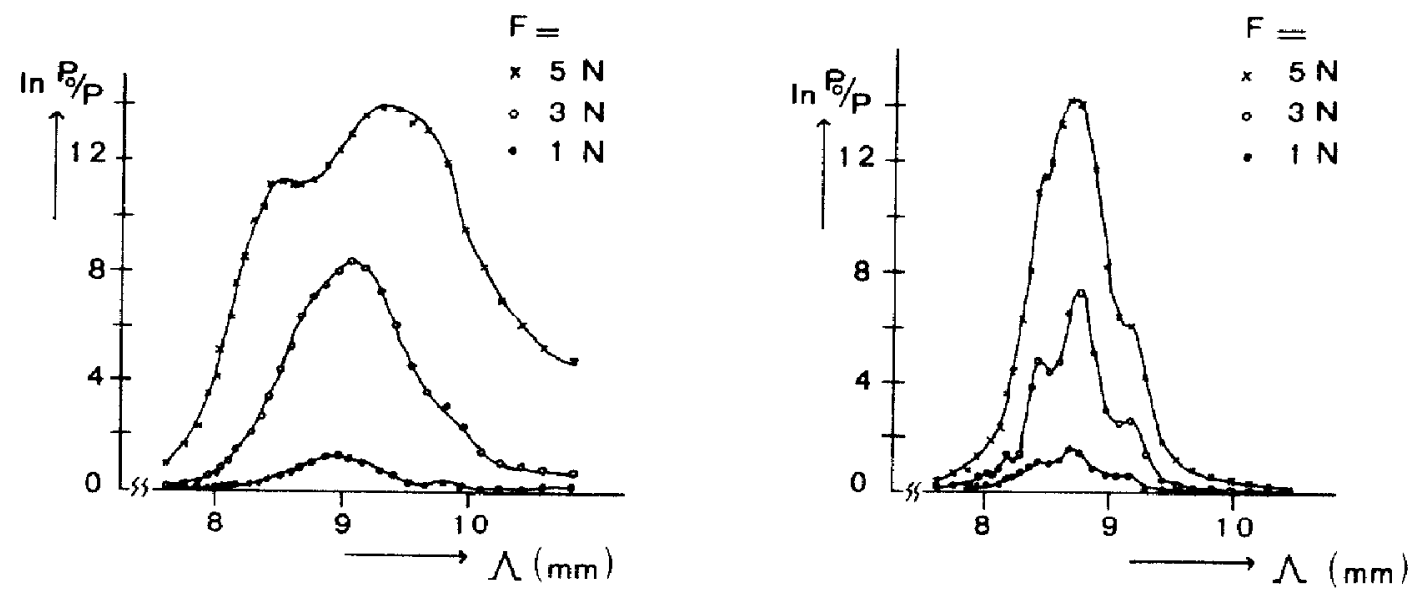

Fig 8 Attenuation versus spatial wavelength at three values of the applied force $N=8$

Fig 9 Attenuation versus spatial wavelength at three values of the applied force $N=28$, second measurement

ridges at three different applied forces Figures 6 to 9 show some results for $28,16,8$ and 28 ridges again, respectively If we compare these results and the results which we obtained with earlier measurements (see [3]), we can make some general statements about this relationship

1) The experimental results show clearly, in agreement with theory, that the spectral width increases with a decreasing number of ridges This holds for all the experiments with $4,8,12,16,20,24$ and 28 ndges respectively

2) It appears that the value of $\Lambda$ for maximum attenuation $\left(=\Lambda_{\max }\right)$ is slightly dependent on the number of ridges This is not in agreement with theory We have not investigated this in more detall yet, and for the time being identify $\Lambda_{c}$ with $\Lambda_{\max }$ 
3) The side maxima occur for all measurements

4) From Figs 6 and 9 it can be seen that the occurrence of the side maxima is not reproducible However, it can be calculated that the distance of these maxima along the $\Lambda$ axis corresponds to the behaviour of eqn (4) quite well

From these results only one valid conclusion can be made the spectral width increases with decreasing number of ridges The occurrence of side maxima and the fact that we cannot reproduce them exactly require further research

The results demonstrate the improvement of our measuring device in earlier experiments we were unable to detect any maximum for a large number of ridges

The second series of measurements concerned the relationship between applied force and attenuation, for all numbers of ridges Figures 10 to 13 show some results Our conclusions from this experiment are summarized as follows

1) In agreement with the given theoretical explanation, it appears that for $\Lambda=\Lambda_{c}$ the relation between force and induced attenuation is quadratic for low, and linear for high values of the force

2) The results are quite similar for all the numbers of ndges The attenuation value where the relation turns over from quadratic into linear is about the same for all experiments

3) The relation depends on the spatial wavelength, as shown in Fig 13 For other numbers of ridges we obtained simlar deviations for $\Lambda \neq \Lambda_{c}$

4) In contrast with former experiments, hysteresis is practically absent This may be ascribed to the fact that the friction and sticking is greatly reduced in the new measuring device

5) The sensitivity of our system is about $10^{-3} \mathrm{~N}$ Improvement of the light power injected into the fibre and of the electronic detection circuitry is expected to increase the sensitivity by at least a factor of 10 or $10^{2}$
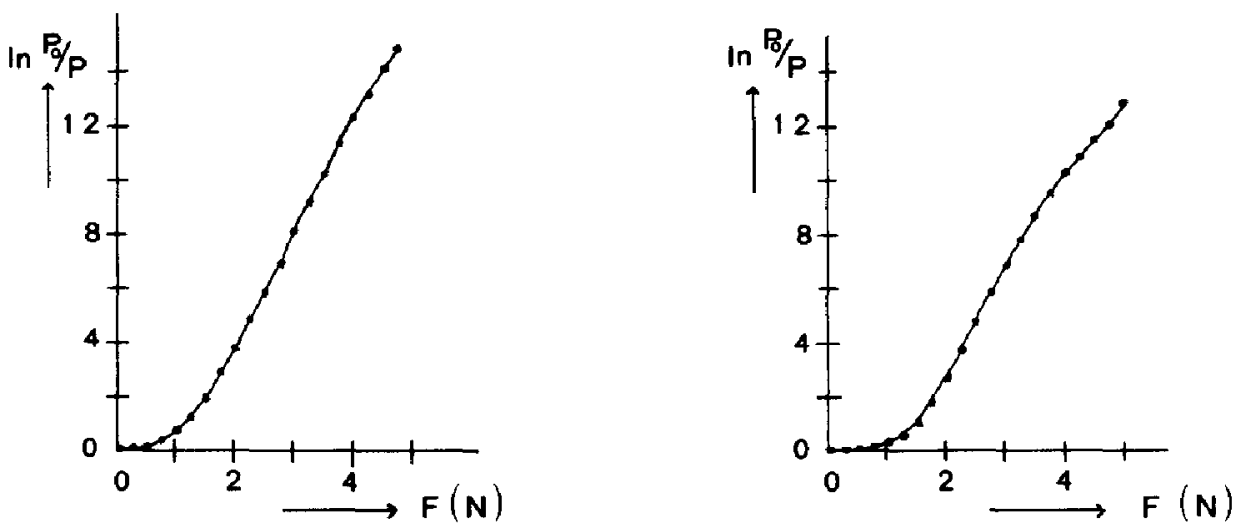

Fig 10 Attenuation versus force $\Lambda=0885 \mathrm{~mm}\left(=\Lambda_{\max }\right), N=28$

Fig 11 Attenuation versus force $\Lambda=0885 \mathrm{~mm}\left(\simeq \Lambda_{\max }\right), N=20$ 

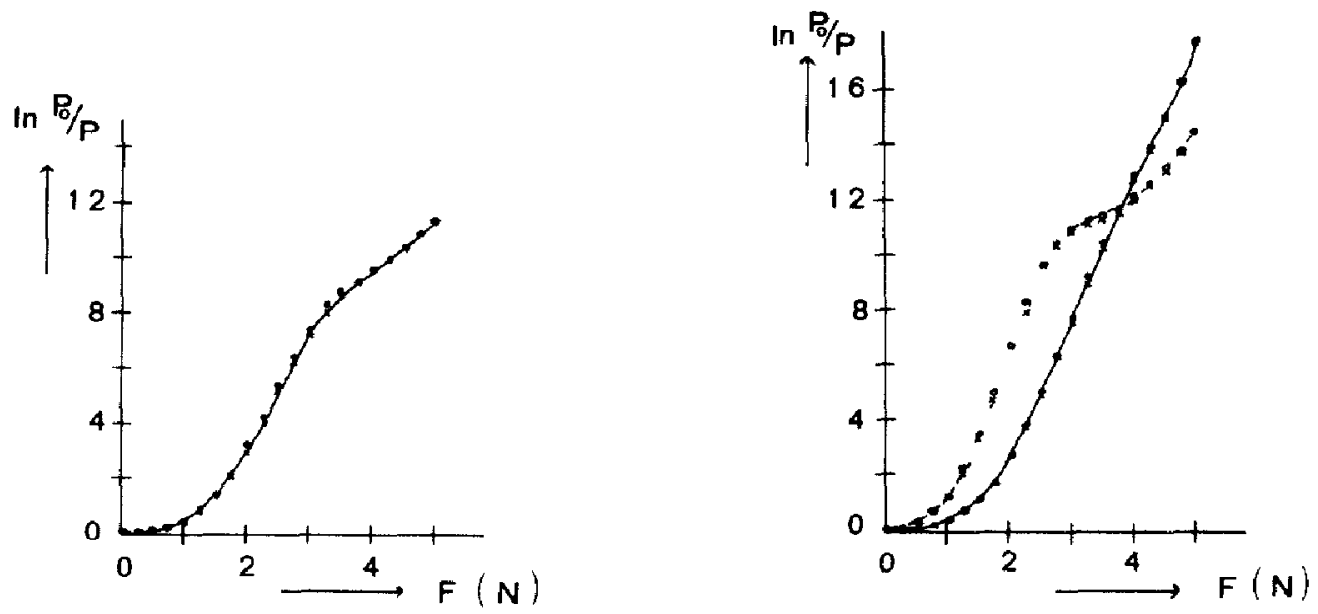

Fig 12 Attenuation versus force $\Lambda=0885 \mathrm{~mm}\left(\neq \Lambda_{\max }\right), N=12$

Fig 13 Attenuation versus force Solıd curve $\Lambda=0935 \mathrm{~mm}\left(=\Lambda_{\max }\right), N=8$ Dotted curve $\Lambda=0885 \mathrm{~mm}\left(\neq \Lambda_{\max }\right), N=8$

6) This sensitivity appears to be rather independent of the number of ridges, again in contrast with our former expenence These newly obtained results are in better agreement with theory

With these experimental results it is possible to check our proposed theoretical prediction For example, from Fig 11 the coefficients of the two equations may be derived as

$\sigma_{1} z=p^{2} F^{2}$ for low values of $F$,

and

$\sigma_{1} z=q F$ for higher values of $F$

It follows that $p^{2} \simeq 008$ and $q \simeq 026$ Substitution of

$F=a d L_{N}$

(with $a$ as a constant of proportionality) in eqn (8) gives

$\sigma_{1} z=\frac{1}{4} \sqrt{\beta C} \frac{F}{a}$

Comparison of eqns (12) and (14) gives

$q=\frac{1}{4 a} \sqrt{\beta C} \simeq 026$

Substitution of eqn (13) in eqn (9) gives

$\sigma_{1} z=\frac{C}{4 a^{2}} F^{2}$

Comparison of eqns (12) and (16) with $p^{2} \simeq 008$ leads to 
$\frac{C}{4 a^{2}} \simeq 008$

hence

$C^{1 / 2} / 2 a \simeq 028$

Substitution of this value in eqn (15) finally gives

$\left(\frac{\beta}{4}\right)^{1 / 2} \simeq 1$

This means that the turmover point of eqn (10) is expected to be found at an attenuation coefficient of about 1 This is a reasonable value in the first place, and is indeed shown by the expernmental results This result is easily obtained for the other experiments performed So in spite of the rather simple approach, the experimental results are in surprising agreement with the theoretical explanation

\section{Conclusions}

The general behaviour of the attenuation coefficient of a fibre which undergoes microbending is pretty well understood However, with respect to, for example, the fine structure of the relation between spatial wavelength and attenuation, more research has to be done to get a better understanding The measured relation between attenuation and applied force at $\Lambda=\Lambda_{c}$ appears to be in a good agreement with our proposed theoretical approach

The influence of the number of applied bends is concentrated on the required accuracy in the manufacture of the ridged profiles Systems with a high number of bends have to be made with a higher accuracy than systems with a low number of bends This is necessary to maintain a good sensitivity and a known relation between applied force and attenuation

We are of the opinion that our results show that microbend-induced attenuation is suited for sensor applications This expectation is supported by the results we obtaned with a prototype load cell based on microbending This prototype had a range of $0-10 \mathrm{~N}$, and the measured non-linearity and hysteresis were less than $1 \%$

\section{Acknowledgements}

The authors wish to thank A J F Hollink and A Bakker for design and manufacturnng of the measurng device, and B Hurenkamp for finıshing the ridged plates and the interference glasses 


\section{References}

$1 \mathrm{D} H$ McMahon, A $\mathrm{R}$ Nelson and W B Spllman Jr, Fibre optic transducers, IEEE Spectrum, 18 (12) (1981) $24-29$

$2 \mathrm{~J} \mathrm{~N}$ Fields and J H Cole, Fibre microbend acoustic sensor, Appl Opt, 19 (19), (1980) $3265-3267$

3 W $\quad H \quad G$ Horsthuss and J H J Fluitman, Sensitivity dependence on number of bends in a fibre optic microbend pressure sensor, $N T G$ Fachberichte Band 79, VDE Verlag GmbH, Berlin, 1982

4 D Marcuse, Theory of Dielectric Optical Waveguides, Academic Press, New York and London, 1974

5 J N Fields, Attenuation of a parabolic index fibre with periodic bends, Appl Phys Lett, $36(10)(1980) 799-801$

6 D Marcuse, Losses and impulse responses of a parabolic index fibre with random bends, Bell Syst Techn J,52(10)(1973) $1423 \quad 1432$

\section{Biographies}

$J H J$ Fluitman was born in Beverwijk, The Netherlands, on March 23, 1938 He recelved his $M$ Sc degree in Experimental Physics in 1966 and a $\mathrm{Ph} \mathrm{D}$ in 1970 from the University of Amsterdam Since 1970 he has been at the Twente University of Technology, Enschede, The Netherlands His research interests include magnetic recording microtransducers for the electrical/mechanical domain and more generally the system aspects of the physical signal conversion process

W $H G$ Horsthuts was born in 1956 in Meppel, The Netherlands From 1974 he studied at Twente University of Technology, where he recelved his B Sc in Electrical Engineering in 1980 Since 1980 he has been in the Sensors and Actuators Research Unit at Twente University, where he is working especially on fibre optic sensors 